\title{
Fuzzy Local Trend Transform based Fuzzy Time Series Forecasting Model
}

\author{
J. Dan, F. Dong, K. Hirota
}

\author{
Jingpei Dan, Fangyan Dong, Kaoru Hirota \\ Tokyo Institute of Technology \\ Japan, 226-8502 Yokohama, 4259 Nagatsuta, Midori-ku, \\ E-mail: \{dan,tou,hirota\}@hrt.dis.titech.ac.jp

\section{Jingpei Dan} \\ Chongqing University \\ P.R.China, 400044 Chongqing, 174 Shazhengjie, Shapingba \\ E-mail: danjingpei@hotmail.com
}

\begin{abstract}
A fuzzy local trend transform based fuzzy time series forecasting model is proposed to improve practicability and forecast accuracy by providing forecast of local trend variation based on the linguistic representation of ratios between any two consecutive points in original time series. Local trend variation satisfies a wide range of real applications for the forecast, the practicability is thereby improved. Specific values based on the forecasted local trend variations that reflect fluctuations in historical data are calculated accordingly to enhance the forecast accuracy. Compared with conventional models, the proposed model is validated by about $50 \%$ and $60 \%$ average improvement in terms of MLTE (mean local trend error) and RMSE (root mean squared error), respectively, for three typical forecasting applications. The MLTE results indicate that the proposed model outperforms conventional models significantly in reflecting fluctuations in historical data, and the improved RMSE results confirm an inherent enhancement of reflection of fluctuations in historical data and hence a better forecast accuracy. The potential applications of the proposed fuzzy local trend transform include time series clustering, classification, and indexing.
\end{abstract}

Keywords: time series forecasting, fuzzy time series, trend, transform.

\section{Introduction}

Based on Zadeh's works (see [1] and [2]), the concept of fuzzy time series and its models for forecasting have been proposed to solve the forecasting problems where the historical data are linguistic values (see [3]- [5]). Conventional fuzzy time series forecasting models that are based on fuzzy time series of original data are limited to forecasting specific values that do not reflect fluctuations in historical data. Local trend variations, however, are mainly concerned with real applications. For example, forecast of changing direction of stock price are more important for stock investors to make reasonable determinations than specific forecast values of stock price. In addition, the forecasted specific demand values are unreliable since historical data is distorted when it transfers along the supply chain due to the bullwhip effect, so local trend variations of demand that are not suffered from the bullwhip effect are more valuable and practical for supply chain managers. Therefore the practicability of conventional fuzzy time series forecasting methods suffers from the limitation of forecasting specific values. This study aims to improve the 
practicability and forecast accuracy by forecasting local trend variations that reflect fluctuations in historical data. It should be noted that the word "trend" usually refers to the long-term trend in statistics, whereas as used in this paper word "trend" means local trend variation in short term or during one period.

Recently, some trend involved fuzzy time series models have been proposed to improve forecasting. Huarng has proposed heuristic models by integrating problem-specific heuristic knowledge with Chen's model [6] to improve forecasting by reflecting the fluctuations in fuzzy time series [7]. A trend-weighted fuzzy time series model for forecasting Taiwan Stock Exchange Capitalization Weighted Stock Index (TAIEX) has been proposed in [8]. Chen and Wang have proposed a method to predict TAIEX based on fuzzy-trend logical relationship groups to improve forecast accuracy [9]. The interval rearranged method has been proposed to reflect fluctuations in historical data and improve forecast accuracy of fuzzy time series in [10]. Although all these methods are involved with trends, they are intrinsically conventional fuzzy time series forecasting methods since they are all based on original data and their forecasting targets are specific values. Fuzzy local trend transform is proposed to provide a different forecasting basis by transforming original data into a linguistic representation of local trend variations called fuzzy local trend time series. In contrast to conventional fuzzy time series forecasting models, local trend variations are forecasted based on the transformed fuzzy local trend time series in the proposed model. Forecast accuracy of specific values is hence enhanced by forecasted local trend variations that reflect fluctuations in historical data.

Three typical forecasting targets, enrollment forecasting, stock index forecasting, and inventory demand forecasting are used to validate the proposed model. To make an effective evaluation, forecasts are evaluated by two measures from different aspects. Root mean squared error (RMSE) is used to evaluate forecast accuracy of specific values while mean local trend error (MLTE) measure [11] is used to evaluate how accurately forecast reflects fluctuations in historical data. For enrollment forecasting, the proposed model outperforms typical fuzzy time series models in terms of RMSE and MLTE. Especially, comparing to Chen's model [6], Huarng's model [12], and Cheng et al.' model [13], MLTE results show an improvement of $73.3 \%, 55.6 \%$, and $66.7 \%$, respectively. For TAIEX forecasting, the proposed model gets the smallest RMSE result while the second rank in terms of MLTE compared to Chen's model [6], Yu's model [14], and Cheng's model [8]. For inventory demand forecasting, compared to Huarng and Yu' model [15], Cheng et al.' model [13], and Chen and Wang' model [9], the proposed model yields about $50 \%, 50 \%$, and $33.3 \%$ improvement in terms of MLTE and $48.3 \%, 73.3 \%$, and $49.1 \%$ improvement in terms of RMSE. The MLTE results demonstrate that the proposed model outperforms conventional fuzzy time series models in significantly reflecting fluctuations in historical data, and the improved RMSE results confirm an inherent enhancement of reflection of fluctuations in historical data and hence a better forecast accuracy.

The rest of the paper is organized as follows: in section 2, fuzzy time series and fuzzy c-means clustering are briefly reviewed. The proposed fuzzy local trend transform based fuzzy time series forecasting model is elaborated in section 3. Empirical analyses on three forecasting targets to demonstrate the proposed model are illustrated in section 4.

\section{Fuzzy Time Series and Fuzzy C-means Clustering: a Brief Re- view}

The proposed model is based on conventional fuzzy time series forecasting model and the fuzzy c-means clustering method is applied in the proposed fuzzy local trend transform, so fuzzy time series and fuzzy c-means clustering are briefly reviewed by adjusting the notations. 


\subsection{Fuzzy time series}

Let $U$ be the universe of discourse, where $U=\left\{u_{1}, u_{2}, \cdots, u_{b}\right\}$. A fuzzy set $A_{i}$ of $U$ is defined as $A_{i}=\sum_{j=1}^{b} f_{A_{i}}\left(u_{j}\right) / u_{j}$, where $f_{A_{i}}$ is the membership function of the fuzzy set $A_{i} ; f_{A_{i}}: U \rightarrow$ $[0,1], u_{a}$ is a generic element of fuzzy set $A_{i}$ and $f_{A_{i}}\left(u_{a}\right)$ is the degree of belongingness of $u_{a}$ to $A_{i} ; f_{A_{i}}\left(u_{a}\right) \in[0,1]$ and $a \in[1, b]$. In [3], the general definitions of fuzzy time series are given as follows:

Definition 1. Let a subset of real numbers $Y(t)(t=\cdots, 0,1,2, \cdots)$ be the universe of discourse by which fuzzy sets $f_{j}(t)$ are defined. If $F(t)$ is a collection of $f_{1}(t), f_{2}(t), \cdots$, then $F(t)$ is called a fuzzy time series defined on $Y(t)$.

Definition 2. If fuzzy time series relationships assume that $F(t)$ is caused only by $F(t-1)$, then the relationship can be expressed as: $F(t)=F(t-1) * R(t, t-1)$, which is the fuzzy relationship between $F(t)$ and $F(t-1)$, where $*$ represents as an operator. (Note that the operator can be either max-min [4], min-max [5], or arithmetic operator [6].)

To sum up, let $F(t-1)=A_{i}$, and $F(t)=A_{j}$, the fuzzy logical relationship between $F(t)$ and $F(t-1)$ can be denoted as $A_{i} \rightarrow A_{j}$, where $A_{i}$ refers to the left-hand side and $A_{j}$ refers to the right-hand side of the FLR. Furthermore, these fuzzy logical relationships can be grouped to establish different fuzzy relationship. These groups are called fuzzy logical relationship groups (FLRGs). On account of its simplicity, FLR method is chosen by most researchers.

The procedure for forecasting using conventional fuzzy time series models has four main steps: (1) Define universe of discourse and intervals; (2) Define fuzzy sets and fuzzify observations in the original time series; (3) Establish fuzzy relationships; (4) Forecast and defuzzify the outcome. Assume that the current state of $F(t)$ is $A_{i}, F_{d e f}(t+1)$ can be forecasted and defuzzified by the following rules:

Rule 1: If there is $A_{i} \rightarrow A_{j}$ in fuzzy logical relationship groups, then $F(t+1)=A_{j}$ and defuzzified as $F_{\text {def }}(t+1)=$ center $_{j}$, where center $_{j}$ is the center of cluster $j$ to which $A_{j}$ belongs.

Rule 2: If there is $A_{i} \rightarrow$ \# in fuzzy logical relationship groups, then $F(t+1)=A_{i}$ and defuzzified as $F_{\text {def }}(t+1)=$ center $_{i}$, where \# represents null value and center $_{i}$ is the center of cluster $i$ to which $A_{i}$ belongs.

Rule 3: If there is $A_{i} A_{1}, A_{2}, \cdots, A_{j}$ in fuzzy logical relationship groups, then the forecast at $t+1$ is calculated as $F_{\text {def }}(t+1)=\left(\right.$ center $_{1}+$ center $_{2}+\cdots+$ center $\left._{j}\right) / j$, where $c_{j}$ is the center of cluster to which $A_{j}$ belongs.

\section{$2.2 \quad$ Fuzzy c-means clustering}

Fuzzy c-means (FCM) clustering is a method of clustering which allows one piece of data to belong to two or more clusters, and is frequently used in pattern recognition [16].

The FCM is based on minimization of the objective function

$$
J_{m}=\sum_{i=1}^{N} \sum_{j=1}^{C} u_{i j}^{m}\left\|x_{i}-c_{j}\right\|^{2}, 1 \leq m \leq \infty,
$$

where $m$ is any real number greater than $1, u_{i j}$ is the degree of membership of $x_{i}$ in the cluster $j, x_{i}$ is the $i$-th of d-dimensional measured data, $c_{j}$ is the d-dimension center of the cluster, and $\|*\|$ is any norm expressing the similarity between any measured data and the center.

Fuzzy partitioning is carried out through an iterative optimization of the objective function (1), with the update of membership $u_{i j}=1 / \sum_{k=1}^{C}\left(\left\|x_{i}-c_{j}\right\| /\left\|x_{i}-c_{k}\right\|\right)^{2 / m-1}$ and the cluster centers $c_{j}=\sum_{i=1}^{N} u_{i j}^{m}\left(x_{i} / \sum_{i=1}^{N} u_{i j}^{m}\right)$. 
This iteration will stop when $\operatorname{Max}_{i j}\left|u_{i j}^{k+1}-u_{i j}^{k}\right|<\varepsilon$, where $\varepsilon$ is a termination criterion between 0 and 1 , whereas $k$ is the iteration step. This procedure converges to a local minimum or a saddle point of $J_{m}$.

\section{Proposed Fuzzy Local Trend Transform based Fuzzy Time Se- ries Forecasting Model}

In literature, trends are widely represented by using absolute variations, slopes, or relative variations between two consecutive points in literature. In section 3.1, trends or local trend variations in study are defined as relative variations, or ratios. The algorithm of fuzzy local trend transform based on local trend variations is then elaborated in section 3.2. The fuzzy time series forecasting model based on fuzzy local trend transform is presented in section 3.3.

\subsection{Local trend variations}

To address the limitation of absolute variations [17] and slopes( [18]- [20])for representing local trend variations, relative variations, which are the ratios between two consecutive data points in a given historical time series, are adopted to indicate local trend variations in this study.

Assuming that for any given time series $P(t), t=1,2, \cdots, n, n \in N$, local trend variation between time $t$ and $t-1$ is defined as

$$
r_{t}=(P(t)-P(t-1)) / P(t-1), t=2,3, \cdots, n,
$$

then the time series of local trend variations for $P(t)$ is defined as $T(t)=r_{t}, t=2, \cdots, n$.

The reasons for forecasting based on local trend variations instead of original time series data are explained as follows. First, the forecasts based on original time series data may not reflect the fluctuations in historical data properly. In most previous studies, the forecasts are equal at some consecutive points which indicated that forecasting based on original time series are not appropriate for reflecting fluctuations [15].

Second, original time series data varies dramatically in different contexts while the time series of local trend variations varies slightly.

Finally, forecasting based on local trend variations are more suitable for reflecting fluctuations in historical data since directions and variation degrees of local trend variations can be indicated by signs and magnitudes of ratios easily. Ratios are preferable in terms of demonstrating the differences in various contexts, ratios-based lengths of intervals are hence adopted to improve fuzzy time series in [15].

Forecasting based on local trend variations are therefore considered more suitable for reflecting fluctuations in historical data and forecast accuracy should be further improved inherently.

\subsection{Fuzzy local trend transform}

To forecast local trend variations, original time series is represented by linguistic local trend variations of original data firstly, the algorithm of fuzzy local trend transform is hence proposed as follows:

Step 1: Obtain the local trend variation time series $T(t)$ by calculating ratios between each two consecutive data points in the original time series $P(t)$ in terms of equation (2).

Step 2: Divide $T(t)$ into three basic clusters in terms of local trend changing direction, i.e., decreasing cluster $T_{d}$, unchanged cluster $T_{u}$, and increasing cluster $T_{i}$. It is easy to determine $T_{d}$ and $T_{i}$ in terms of sign of ratios after $T_{u}$ is determined. Assume that the interval for unchanged 
Table 1: Parameter ŚÁ for determining the interval of unchanged cluster

\begin{tabular}{|c|c|c|c|c|}
\hline $\operatorname{Max}(|T(t)|)\left(\times 10^{-2}\right)$ & $\operatorname{Max}(|T(t)|) \leq 1$ & $\operatorname{Max}(|T(t)|) \leq 10$ & $\operatorname{Max}(|T(t)|) \leq 20$ & $\cdots$ \\
\hline$\alpha\left(\times 10^{-2}\right)$ & 0.01 & 0.1 & 0.2 & $\cdots$ \\
\hline
\end{tabular}

cluster is $[-\alpha, \alpha], \alpha$ is determined by $\operatorname{Max}(|T(t)|)$ according to Table 1 since it is possible that the definition of unchanged cluster varies from problem to problem. The observations in $T(t)$, of which the values are greater than $\alpha$, are then assigned to $T_{i}$, while the observations of which the values less than $-\alpha$ are assigned to $T_{d}$.

Step 3: Divide $T_{i}$ and $T_{d}$ into $c_{i}$ and $c_{d}$ clusters by applying FCM, respectively. Assume that the number of observations in $T_{i}$ and $T_{d}$ are $n_{i}$ and $n_{d}$, respectively, then the number of clusters for $T_{i}, T_{d}$ and $T(t)$ are predefined by users as $c_{i}\left(2 \leq c_{i} \leq n_{i}\right), c_{d}\left(2 \leq c_{d} \leq n_{d}\right)$, and $c\left(c=c_{d}+c_{i}+1\right)$, respectively. $T_{i}$ and $T_{d}$ are then divided into $c_{i}$ and $c_{d}$ clusters by FCM, respectively, as described in section 2.2. Consequently, the cluster centers and memberships with respect to the clusters are obtained.

Step 4: Fuzzify the local trend time series $T(t)$ as fuzzy local trend time series $F_{T}(t)$. First, the clusters of $T(t)$ are achieved by combining the clustering results obtained in Step 2 and Step 3. Then the linguistic terms $A_{i}(i=1,2, \cdots, c)$ are defined corresponding to the clusters. $T(t)$ is finally fuzzified into $F_{T}(t)$ by assigning $A_{i}$ to $T(t)$ when the maximum membership of $T(t)$ occurs at the cluster to which $A_{i}$ belongs.

\subsection{Fuzzy local trend transform based fuzzy time series forecasting model}

Theoretically, the proposed fuzzy local trend transform can be integrated with any conventional fuzzy time series forecasting model. Because of simplicity, the proposed model is integrated with Chen's model [6] as stated in section 2.1. The proposed model differs from Chen' model in Step 1 and Step 4 as described in the following:

Step 1: Transform original time series into fuzzy local trend time series by the proposed fuzzy local trend transform as stated in section 3.2.

Step 2: Establish fuzzy logical relationships and fuzzy logical relationship groups based on fuzzy local trend time series obtained in Step 1 as described in section 2.1.

Step 3: Forecast and defuzzify the possible outcomes of local trend variations, which is denoted as $T_{\text {def }}(t), t=1,2, \cdots, n-1$, based on fuzzy logical relationship groups as described in section 2.1.

Step 4: Calculate specific values based on forecasted local trend variations obtained in Step 3 in terms of equation(3) that is defined as

$$
P_{\text {pre }}(t)=P(t) \times T_{\text {def }}(t), t=1,2, \cdots, n-1,
$$

where $P_{\text {pre }}(t)$ indicates predicted specific values.

\section{Empirical Analyses on Forecasting based on Fuzzy Local Trend Time Series}

To validate the proposed model, three applications are used in the empirical analyses, including student enrollment forecasting (the enrollments of the University of Alabama [4]), stock index forecasting (Taiwan Stock Exchange Capitalization Weighted Stock Index (TAIEX)), and inventory demand forecasting [15]. The first two data sets are algebraic growth data and widely used to validate fuzzy time series models in many relevant studies while the inventory demand data set is exponential growth data and typical in supply chain management application. We 
compare the proposed model with typical fuzzy time series models in terms of two forecast accuracy measures. One is conventional measure RMSE (root mean squared error) that is commonly used to measure forecast accuracy of specific values based on quantitative error in fuzzy time series forecasting, while the other is MLTE (mean local trend error), which is proposed to measure how accurately forecasts reflect fluctuations in actual data based on local trend error [11]. It is more effective and proper for comparing the models by evaluating them from two different aspects than using one or more conventional measures that based on quantitative error. For a given time series $y_{t}, t=1, \cdots, n$, the prediction of $y_{t}$ is $f_{t}, t=1, \cdots, n$, RMSE is defined as

$$
R M S E=\sqrt{\frac{1}{n} \sum_{t=1}^{n}\left(f_{t}-y_{t}\right)^{2}}, t=1,2, \cdots, n, n \in N,
$$

AzA while MLTE is defined as

$M L T E=\frac{1}{n-1} \sum_{i=1}^{n-1} E_{i} \times 100 \%, \quad E_{i}=\left\{\begin{array}{l}1 \operatorname{sign}\left(y_{t+1}-y_{t}\right) \neq \operatorname{sign}\left(f_{t+1}-f_{t}\right) \\ 0 \operatorname{sign}\left(y_{t+1}-y_{t}\right)=\operatorname{sign}\left(f_{t+1}-f_{t}\right)\end{array} \quad t=1,2, \cdots, n, n \in N\right.$,

where $E_{i}$ indicates the number of local trend change errors, sign indicates the operator for outputting the sign of the operand. When the predicted local trend variation is inconsistent with the original one in the same interval, $E_{i}$ is equal to 1 , otherwise $E_{i}$ equals to 0 [11].

The experiments are implemented using Matlab R2010a. In section 4.1, the forecasting algorithm is illustrated step by step with the example of enrollment forecasting. Performance of the proposed model for stock index and inventory demand forecasting are analyzed in section 4.2 and section 4.3 , respectively.

\subsection{Forecasting enrollments}

The yearly data of student enrollments of the University of Alabama from 1971 to 1992 are commonly used in previous studies on fuzzy time series to validate fuzzy time series models. To make proper comparison with other models, the same data set is used in this study to validate the proposed model. The procedure of forecasting enrollments by the proposed model is as follows:

Step 1: Transform original time series into fuzzy local trend time series by the proposed fuzzy local trend transform.

(1) Obtain the local trend time series $T(t)$ as shown in the third column of Table 2 by calculating the local trend variations of the enrollments between each two consecutive years in the original time series $P(t)$ in terms of equation(2).

(2) Divide $T(t)$ into decreasing cluster $T_{d}$, unchanged cluster $T_{u}$, and increasing cluster $T_{i}$. Since none of the observations in $T(t)$ is equal to 0 and maximum absolute value of the observations in $T(t)$ is $7.6675 \%, \alpha$ is determined to be $0.1 \%$ according to Table 1 . Consequently, $T_{d}=\{-5.8274,-3.1385,-2.3840,-2.2714,-0.9638\}, T_{u}=\{0.0466\}$, and $T_{i}=\{0.1189,0.4147$, $0.6664,1.6535,1.8872,1.9071,2.2414,3.8912,4.5179,5.1987,5.4145,5.4742,5.9643,5.9782$, $7.6576\}$.

(3) Divide $T_{i}$ and $T_{d}$ into $c_{i}$ into $c_{d}$ clusters by applying FCM, respectively. For FCM, the predefined number of clusters $c=c_{d}+c_{i}+1$. To make fair comparison, the total number of clusters is predefined to be 7 in this study as the same as used in the previous studies. Assume that $c_{d}=c_{i}=3$, the cluster centers of $T_{d}$ and $T_{i}$ and the membership grades of $T_{d}$ and $T_{i}$ are shown in Table 3 and Table 4, respectively. Naturally, the center of unchanged cluster $T_{i}$ is $0 \%$.

(4) Fuzzify the local trend time series $T(t)$ into fuzzy local trend time series $F_{T}(t)$ as shown in the fourth column of Table 2. Combine the clustering results obtained in Step (2) and Step 
Fuzzy Local Trend Transform based Fuzzy Time Series Forecasting Model

Table 2: Forecasting local trend variations and specific values of the enrollments

\begin{tabular}{|c|c|c|c|c|c|}
\hline Year & $\begin{array}{c}\text { Actual } \\
\text { enrollment }\end{array}$ & $\begin{array}{l}\text { Local trend } \\
\text { variation }\end{array}$ & $\begin{array}{l}\text { Fuzzified local } \\
\text { trend variation }\end{array}$ & $\begin{array}{l}\text { Forecasted local trend } \\
\text { variation }\left(\times 10^{-2}\right)\end{array}$ & $\begin{array}{l}\text { Forecasted } \\
\text { enrollment }\end{array}$ \\
\hline 1971 & 13055 & - & - & - & - \\
\hline 1972 & 13563 & 3.8912 & A6 & - & - \\
\hline 1973 & 13867 & 2.2414 & A5 & 0.1233 & 13580 \\
\hline 1974 & 14696 & 5.9782 & A7 & 1.1623 & 14028 \\
\hline 1975 & 15460 & 5.1987 & A6 & 3.8752 & 15266 \\
\hline 1976 & 15311 & -0.96378 & A3 & 0.1233 & 15479 \\
\hline 1977 & 15603 & 1.9071 & $\overline{\mathrm{A} 5}$ & 1.2224 & 15498 \\
\hline 1978 & 15861 & 1.6535 & A5 & 1.1623 & 15784 \\
\hline 1979 & 16807 & 5.9643 & A7 & 1.1623 & 16045 \\
\hline 1980 & 16919 & 0.66639 & $\overline{\mathrm{A} 5}$ & 3.8752 & 17458 \\
\hline 1981 & 16388 & -3.1385 & $\mathrm{~A} 2$ & 1.1623 & 17116 \\
\hline 1982 & 15433 & -5.8274 & $\mathrm{~A} 1$ & -2.3003 & 16011 \\
\hline 1983 & 15497 & 0.4147 & A5 & 1.2224 & 15622 \\
\hline 1984 & 15145 & -2.2714 & $\mathrm{~A} 2$ & 1.1623 & 15677 \\
\hline 1985 & 15163 & 0.11885 & A5 & -2.3003 & 14797 \\
\hline 1986 & 15984 & 5.4145 & A7 & 1.1623 & 15339 \\
\hline 1987 & 16859 & 5.4742 & A7 & 3.8752 & 16603 \\
\hline 1988 & 18150 & 7.6576 & A7 & 3.8752 & 17512 \\
\hline 1989 & 18970 & 4.5179 & A6 & 3.8752 & 18853 \\
\hline 1990 & 19328 & 1.8572 & A5 & 0.1233 & 18993 \\
\hline 1991 & 19337 & 0.046565 & A4 & 1.1623 & 19553 \\
\hline 1992 & 18876 & -2.384 & A2 & -2.5770 & 18839 \\
\hline RMSE & & & & & 438.18 \\
\hline MLTE & & & & & $21.0526 \%$ \\
\hline
\end{tabular}

(3) to achieve the clusters of $T(t)$, the linguistic variables are then defined as shown in Table 5 according to the clusters. Each local trend variation in $T(t)$ is fuzzified by the linguistic variable to which the maximum membership belongs in terms of the results in Table 3 and Table 4.

Table 3: Membership grades of decreasing cluster for each linguistic variable

\begin{tabular}{|c|c|c|c|c|c|c|c|}
\hline clusters & Cluster Centers & $\begin{array}{c}\text { Linguistic } \\
\text { variables }\end{array}$ & $T_{d}(1)$ & $T_{d}(2)$ & $T_{d}(3)$ & $T_{d}(4)$ & $T_{d}(5)$ \\
\hline 1 & -2.5770 & $\mathrm{~A} 2$ & 0.0000018 & 0.9 & 0.9785 & 0.9407 & 0.000055 \\
\hline 2 & -5.8231 & $\mathrm{~A} 1$ & 0.9999974 & 0.0393 & 0.0031 & 0.0070 & 0.000006 \\
\hline 3 & -0.9758 & $\mathrm{~A} 3$ & 0.0000008 & 0.0607 & 0.0184 & 0.0523 & 0.999939 \\
\hline & & $F_{T}(t)$ & $\mathrm{A} 1$ & $\mathrm{~A} 2$ & $\mathrm{~A} 2$ & $\mathrm{~A} 2$ & $\mathrm{~A} 3$ \\
\hline
\end{tabular}

Step 2: The FLRs of $F_{T}(t)$ are established as shown in Table 6 according to Definition 3 in 2.1. Then, the FLRs are rearranged into FLRGs as shown in Table 7.

Step 3: The possible outcomes of local trend variations from 1973 to 1992 are forecasted and defuzzified as shown in the fifth column of Table 2.

Table 4: Membership grades of increasing cluster for each linguistic variable

\begin{tabular}{|c|c|c|c|c|c|c|c|c|c|c|}
\hline clusters & $\begin{array}{c}\text { Cluster } \\
\text { Centers }\end{array}$ & $\begin{array}{c}\text { Linguistic } \\
\text { variables }\end{array}$ & $T_{i}(1)$ & $T_{i}(2)$ & $T_{i}(3)$ & $T_{i}(4)$ & $T_{i}(5)$ & $T_{i}(6)$ & $T_{i}(7)$ & $T_{i}(8)$ \\
\hline 1 & 4.3997 & $\mathrm{~A} 6$ & 0.0603 & 0.0387 & 0.0215 & 0.0238 & 0.0639 & 0.0684 & 0.1719 & 0.9139 \\
\hline 2 & 1.2224 & $\mathrm{~A} 5$ & 0.9077 & 0.9416 & 0.9680 & 0.9667 & 0.9123 & 0.9063 & 0.7715 & 0.0332 \\
\hline 3 & 6.0036 & $\mathrm{~A} 7$ & 0.0319 & 0.0197 & 0.0105 & 0.0095 & 0.0238 & 0.0253 & 0.0566 & 0.0530 \\
\hline & & $F_{T}(t)$ & $\mathrm{A} 5$ & $\mathrm{~A} 5$ & $\mathrm{~A} 5$ & $\mathrm{~A} 5$ & $\mathrm{~A} 5$ & $\mathrm{~A} 5$ & $\mathrm{~A} 5$ & $\mathrm{~A} 6$ \\
\hline & & & $T_{i}(9)$ & $T_{i}(10)$ & $T_{i}(11)$ & $T_{i}(12)$ & $T_{i}(13)$ & $T_{i}(14)$ & $T_{i}(15)$ & \\
\hline 1 & 4.3997 & $\mathrm{~A} 6$ & 0.9924 & 0.4936 & 0.2486 & 0.1929 & 0.00063 & 0.00027 & 0.1947 & \\
\hline 2 & 1.2224 & $\mathrm{~A} 5$ & 0.0013 & 0.0199 & 0.0146 & 0.0123 & 0.00007 & 0.00003 & 0.0499 & \\
\hline 3 & 6.0036 & $\mathrm{~A} 7$ & 0.0063 & 0.4864 & 0.7371 & 0.7948 & 0.9993 & 0.9997 & 0.7554 & \\
\hline & & $F_{T}(t)$ & $\mathrm{A} 6$ & $\mathrm{~A} 6$ & $\mathrm{~A} 7$ & $\mathrm{~A} 7$ & $\mathrm{~A} 7$ & $\mathrm{~A} 7$ & $\mathrm{~A} 7$ & \\
\hline
\end{tabular}

Step 4: The enrollment values from the year 1973 to 1992 are calculated based on the forecasted local trend variations obtained in Step 3 as shown in the last column of Table 2. The number of intervals of the universe of discourse affects forecasting results [21]. When analyzing the sensitivity of $c$, which varies in $\{7,9,11,13\}$, the RMSE and MLTE results $\{502.401,440.3566,380.8953,313.2307$ 
Table 5: Cluster centers of local trend variations of enrollments

\begin{tabular}{|c|l|}
\hline $\begin{array}{c}\text { Cluster Centers } \\
\left(\times 10^{-2}\right)\end{array}$ & Linguistic variables \\
\hline-5.8231 & $\mathrm{~A} 1$ (big decrease) \\
\hline-2.5770 & $\mathrm{~A} 2$ (decrease) \\
\hline-0.9758 & $\mathrm{~A} 3$ (small decrease) \\
\hline 0 & $\mathrm{~A} 4($ almost unchanged $)$ \\
\hline 1.2224 & $\mathrm{~A} 5$ (small increase) \\
\hline 4.3997 & $\mathrm{~A} 6$ (increase) \\
\hline 6.0036 & $\mathrm{~A} 7$ (big increase) \\
\hline
\end{tabular}

and $\{23.1579 \%, 24.2105 \%, 20 \%, 13.6842 \%\}$ respectively. The average RMSE and MLTE results indicate that forecast error decrease as the number of clusters increases, in other words, the bigger the number of clusters the higher the forecast accuracy.

Table 6: Fuzzy logical relationships of local trend variations of enrollments

\begin{tabular}{|llllll|}
\hline $\mathrm{A} 6 \rightarrow \mathrm{A} 5$ & $\mathrm{~A} 5 \rightarrow \mathrm{A} 7$ & $\mathrm{~A} 7 \rightarrow \mathrm{A} 6$ & $\mathrm{~A} 6 \rightarrow \mathrm{A} 3$ & $\mathrm{~A} 3 \rightarrow \mathrm{A} 5$ & \\
\hline $\mathrm{A} 5 \rightarrow \mathrm{A} 5$ & $\mathrm{~A} 5 \rightarrow \mathrm{A} 7$ & $\mathrm{~A} 7 \rightarrow \mathrm{A} 5$ & $\mathrm{~A} 5 \rightarrow \mathrm{A} 2$ & $\mathrm{~A} 2 \rightarrow \mathrm{A} 1$ & \\
\hline $\mathrm{A} 1 \rightarrow \mathrm{A} 5$ & $\mathrm{~A} 5 \rightarrow \mathrm{A} 2$ & $\mathrm{~A} 2 \rightarrow \mathrm{A} 5$ & $\mathrm{~A} 5 \rightarrow \mathrm{A} 7$ & $\mathrm{~A} 7 \rightarrow \mathrm{A} 7$ & \\
\hline $\mathrm{A} 7 \rightarrow \mathrm{A} 7$ & $\mathrm{~A} 7 \rightarrow \mathrm{A} 6$ & $\mathrm{~A} 6 \rightarrow \mathrm{A} 5$ & $\mathrm{~A} 5 \rightarrow \mathrm{A} 4$ & $\mathrm{~A} 4 \rightarrow \mathrm{A} 2$ & $\mathrm{~A} 2 \rightarrow \mathrm{\#}$ \\
\hline
\end{tabular}

Table 7: Fuzzy logical relationship groups of local trend variations of enrollments

\begin{tabular}{|c|c|c|c|}
\hline Group 1: A1 $\rightarrow \mathrm{A} 5$ & Group 2: $\mathrm{A} 2 \rightarrow \mathrm{A} 1, \mathrm{~A} 5, \#$ & Group 3: A3 $\rightarrow$ A5 & Group 4: $\mathrm{A} 4 \rightarrow \mathrm{A} 2$ \\
\hline Group 5: A5 $\rightarrow \mathrm{A} 2$, & Group 6: $\mathrm{A}$ & Group & $5 \Delta 6 \quad \Delta 7$ \\
\hline
\end{tabular}

Table 8: Comparisons for enrollment forecasting under the same conditions

\begin{tabular}{|c|c|c|c|c|c|}
\hline Year & $\begin{array}{c}\text { Actual } \\
\text { enrollment }\end{array}$ & $\begin{array}{c}\text { Chen } \\
{[6]}\end{array}$ & $\begin{array}{c}\text { Huarng } \\
{[12]}\end{array}$ & $\begin{array}{c}\text { Cheng et al. } \\
{[13]}\end{array}$ & $\begin{array}{c}\text { The proposed } \\
\text { model }\end{array}$ \\
\hline 1971 & 13055 & - & - & - & -AzA \\
\hline 1972 & 13563 & 14000 & 14000 & 14242 & -AzA \\
\hline 1973 & 13867 & 14000 & 14000 & 14242 & 13580 \\
\hline 1974 & 14696 & 14000 & 14000 & 14242 & 14028 \\
\hline 1975 & 15460 & 15500 & 15500 & 15474.3 & 15266 \\
\hline 1976 & 15311 & 16000 & 15500 & 15474.3 & 15479 \\
\hline 1977 & 15603 & 16000 & 16000 & 15474.3 & 15498 \\
\hline 1978 & 15861 & 16000 & 16000 & 15474.3 & 15784 \\
\hline 1979 & 16807 & 16000 & 16000 & 16146.5 & 16045 \\
\hline 1980 & 16919 & 16833 & 17500 & 16988.3 & 17458 \\
\hline 1981 & 16388 & 16833 & 16000 & 16988.3 & 17116 \\
\hline 1982 & 15433 & 16833 & 16000 & 16146.5 & 16011 \\
\hline 1983 & 15497 & 16000 & 16000 & 15474.3 & 15622 \\
\hline 1984 & 15145 & 16000 & 15500 & 15474.3 & 15677 \\
\hline 1985 & 15163 & 16000 & 16000 & 15474.3 & 14797 \\
\hline 1986 & 15984 & 16000 & 16000 & 15474.3 & 15339 \\
\hline 1987 & 16859 & 16000 & 16000 & 16146.5 & 16603 \\
\hline 1988 & 18150 & 16833 & 17500 & 16988.3 & 17512 \\
\hline 1989 & 18970 & 19000 & 19000 & 19144 & 18853 \\
\hline 1990 & 19328 & 19000 & 19000 & 19144 & 18993 \\
\hline 1991 & 19337 & 19000 & 19500 & 19144 & 19553 \\
\hline 1992 & 18876 & 19000 & 19000 & 19144 & 18839 \\
\hline RMSE ÅA & & 646.79 & 477.91 & 466.17 & 438.18 \\
\hline MLTE & & $78.9474 \%$ & $47.3684 \%$ & $63.1579 \%$ & $21.0526 \%$ \\
\hline & & & & & \\
\hline
\end{tabular}

The proposed method is compared with typical models for enrollment forecasting, including Chen's model [6], Huarng's model [12], and Cheng et al.' model [13]. To make fair comparison, seven linguistic variables are defined in all the compared models and the RMSEs and MLTEs are computed with reference to the year 1973. As shown in Table 8, the comparative results show that the proposed method outperforms the other models in terms of RMSE and MLTE. 
Especially, comparing to Chen's model, Huarng's model, and Cheng et al.' model, the proposed model makes about $73.3 \%, 55.6 \%$, and $66.7 \%$ improvements in terms of MLTE, respectively.

\subsection{Stock index forecasting}

Table 9: Comparisons for TAIEX forecasting

\begin{tabular}{|c|c|c|c|c|c|c|c|}
\hline Date & $\begin{array}{l}\text { Actual } \\
\text { Index }\end{array}$ & $\begin{array}{c}\text { Actual local } \\
\text { trend }\left(\times 10^{-2}\right)\end{array}$ & $\begin{array}{c}\text { Chen } \\
{[6]}\end{array}$ & $\begin{array}{l}\mathrm{Yu} \\
{[14]}\end{array}$ & $\begin{array}{c}\text { Cheng } \\
{[8]}\end{array}$ & $\begin{array}{c}\text { The proposed } \\
\text { model }\end{array}$ & $\begin{array}{c}\text { Forecasted local } \\
\text { trend }\left(\times 10^{-2}\right)\end{array}$ \\
\hline $00 / 11 / 02$ & $5,626.08$ & 1.477 & 5300 & 5340 & 5463.85 & 5524.81 & -0.35 \\
\hline $00 / 11 / 03$ & $5,796.08$ & 2.933 & 5750 & 5721.67 & 5644.8 & 5611.08 & -0.27 \\
\hline $00 / 11 / 04$ & $5,677.30$ & -2.0922 & 5450 & 5435 & 5797.8 & 5815.39 & 0.33 \\
\hline $00 / 11 / 06$ & $5,657.48$ & -0.35033 & 5750 & 5721.67 & 5690.9 & 5657.47 & -0.35 \\
\hline $00 / 11 / 07$ & $5,877.77$ & 3.7478 & 5750 & 5721.67 & 5673.06 & 5648.99 & -0.15 \\
\hline $00 / 11 / 08$ & $6,067.94$ & 3.134 & 5750 & 5760 & 5871.32 & 5897.36 & 0.33 \\
\hline $00 / 11 / 09$ & $6,089.55$ & 0.35487 & 6075 & 6062.5 & 6042.47 & 6088.09 & 0.33 \\
\hline $00 / 11 / 10$ & $6,088.74$ & -0.013303 & 6075 & 6062.5 & 6061.92 & 6093.84 & 0.07 \\
\hline $00 / 11 / 13$ & $5,793.52$ & -5.0957 & 6075 & 6062.5 & 6061.19 & 6079.54 & -0.15 \\
\hline $00 / 11 / 14$ & $5,772.51$ & -0.36397 & 5450 & 5435 & 5795.5 & 5685.36 & -1.87 \\
\hline $00 / 11 / 15$ & $5,737.02$ & -0.61861 & 5450 & 5435 & 5776.59 & 5763.81 & -0.15 \\
\hline $00 / 11 / 16$ & $5,454.13$ & -5.1867 & 5450 & 5435 & 5744.65 & 5728.37 & -0.15 \\
\hline $00 / 11 / 17$ & $5,351.36$ & -1.9204 & 5300 & 5340 & 5409.92 & 5352.29 & -1.87 \\
\hline $00 / 11 / 18$ & $5,167.35$ & -3.561 & 5350 & 5350 & 5317.42 & 5304.12 & -0.88 \\
\hline $00 / 11 / 20$ & $4,845.21$ & -6.6486 & 5150 & 5150 & 5151.81 & 5070.94 & -1.87 \\
\hline $00 / 11 / 21$ & $5,103.00$ & 5.0517 & 4850 & 4850 & 4861.89 & 4953.18 & 2.23 \\
\hline $00 / 11 / 22$ & $5,130.61$ & 0.53814 & 5150 & 5150 & 5093.9 & 5120.92 & 0.35 \\
\hline $00 / 11 / 23$ & $5,146.92$ & 0.31689 & 5150 & 5150 & 5118.75 & 5134.17 & 0.07 \\
\hline $00 / 11 / 24$ & $5,419.99$ & 5.0382 & 5150 & 5150 & 5213.56 & 5150.48 & 0.07 \\
\hline $00 / 11 / 27$ & $5,433.78$ & 0.25378 & 5300 & 5340 & 5459.32 & 5439.04 & 0.35 \\
\hline $00 / 11 / 28$ & $5,362.26$ & -1.3338 & 5300 & 5340 & 5391.6 & 5425.62 & -0.15 \\
\hline $00 / 11 / 29$ & $5,319.46$ & -0.80459 & 5350 & 5350 & 5327.23 & 5314.92 & -0.88 \\
\hline $00 / 11 / 30$ & $5,256.93$ & -1.1895 & 5350 & 5350 & 5288.71 & 5311.49 & -0.15 \\
\hline $00 / 12 / 01$ & $5,342.06$ & 1.5936 & 5250 & 5250 & 5232.44 & 5210.46 & -0.88 \\
\hline $00 / 12 / 02$ & $5,277.35$ & -1.2262 & 5350 & 5350 & 5309.05 & 5327.84 & -0.27 \\
\hline $00 / 12 / 04$ & $5,174.02$ & -1.9971 & 5250 & 5250 & 5250.81 & 5230.78 & -0.88 \\
\hline $00 / 12 / 05$ & $5,199.20$ & 0.48431 & 5150 & 5150 & 5157.82 & 5128.29 & -0.88 \\
\hline $00 / 12 / 06$ & $5,170.62$ & -0.55274 & 5150 & 5150 & 5180.48 & 5202.82 & 0.07 \\
\hline $00 / 12 / 07$ & $5,212.73$ & 0.80783 & 5150 & 5150 & 5154.76 & 5162.82 & -0.15 \\
\hline $00 / 12 / 08$ & $5,252.83$ & 0.7634 & 5250 & 5250 & 5192.66 & 5216.33 & 0.07 \\
\hline $00 / 12 / 11$ & $5,284.41$ & 0.59761 & 5250 & 5250 & 5228.75 & 5256.46 & 0.07 \\
\hline $00 / 12 / 12$ & $5,380.09$ & 1.7784 & 5250 & 5250 & 5257.17 & 5288.08 & 0.07 \\
\hline $00 / 12 / 13$ & $5,384.36$ & 0.079304 & 5350 & 5350 & 5343.28 & 5398.00 & 0.33 \\
\hline $00 / 12 / 14$ & $5,320.16$ & -1.2067 & 5350 & 5350 & 5347.12 & 5376.30 & -0.15 \\
\hline $00 / 12 / 15$ & $5,224.74$ & -1.8263 & 5350 & 5350 & 5289.34 & 5273.20 & -0.88 \\
\hline $00 / 12 / 16$ & $5,134.10$ & -1.7655 & 5250 & 5250 & 5203.46 & 5178.54 & -0.88 \\
\hline $00 / 12 / 18$ & $5,055.20$ & -1.5608 & 5150 & 5150 & 5121.89 & 5088.74 & -0.88 \\
\hline $00 / 12 / 19$ & $5,040.25$ & -0.29661 & 5450 & 5405 & 5050.88 & 5010.54 & -0.88 \\
\hline $00 / 12 / 20$ & $4,947.89$ & -1.8667 & 5450 & 5405 & 5037.42 & 5032.72 & -0.15 \\
\hline $00 / 12 / 21$ & $4,817.22$ & -2.7126 & 4950 & 4950 & 4954.3 & 4904.19 & -0.88 \\
\hline $00 / 12 / 22$ & $4,811.22$ & -0.12471 & 4850 & 4850 & 4836.7 & 4800.37 & -0.35 \\
\hline $00 / 12 / 26$ & $4,721.36$ & -1.9033 & 4850 & 4850 & 4831.3 & 4803.96 & -0.15 \\
\hline $00 / 12 / 27$ & $4,614.63$ & -2.3129 & 4750 & 4750 & 4750.42 & 4679.69 & -0.88 \\
\hline $00 / 12 / 28$ & $4,797.14$ & 3.8046 & 4650 & 4650 & 4654.37 & 4598.48 & -0.35 \\
\hline $00 / 12 / 29$ & $4,743.94$ & -1.1214 & 4750 & 4750 & 4818.62 & 4813.06 & 0.33 \\
\hline $00 / 12 / 30$ & $4,739.09$ & -0.10234 & 4750 & 4750 & 4770.74 & 4736.76 & -0.15 \\
\hline RMSE & & & 176.32 & 170.27 & 121.47 & 114.63 & \\
\hline MLTE & & & $64.44 \%$ & $62.22 \%$ & $26.67 \%$ & $31.11 \%$ & \\
\hline
\end{tabular}

The daily stock index, TAIEX (Taiwan Stock Exchange Capitalization Weighted Stock Index), which is the other widely used data set in fuzzy time series studies, is used to further validate the proposed model for out-sample forecasting. The TAIEX data during 2000/01/01 $2000 / 10 / 31$ are used as training data set, and the data during 2000/11/01 - 2000/12/31 are used as testing data set. 
The proposed model is compared with Chen's model [6], Yu's model [14], and Cheng's model [8]. The comparison of the forecasting results is shown in Table 9. The proposed model gets the smallest RMSE result while the second rank in terms of MLTE among the compared models. Cheng's model gets the smallest MLTE since it incorporates trend-weighting into Chen's model for TAIEX forecasting. The proposed model, however, improves forecast accuracy of specific value about $6 \%$ compared to Cheng's model by reflecting fluctuations in historical data.

\subsection{Inventory demand forecasting}

Demand forecasting plays a very important role in supply chain management. To further validate the applicability of the proposed method for demand forecasting, an inventory demand data set [15] is used in this study. This data set has been used in several previous studies, so it is proper for fair comparison. Inventory demand data from 1 to 19 are used as training set while data from 20 to 24 are used as testing set.

Table 10: Comparisons for inventory demand forecasting

\begin{tabular}{|c|c|c|c|c|c|}
\hline Time & $\begin{array}{c}\text { Actual } \\
\text { inventory demand }\end{array}$ & $\begin{array}{c}\text { Huarng and Yu } \\
{[15]}\end{array}$ & $\begin{array}{c}\text { Cheng et al. } \\
{[13]}\end{array}$ & $\begin{array}{c}\text { Chen and Wang } \\
{[9]}\end{array}$ & $\begin{array}{c}\text { The proposed } \\
\text { model }\end{array}$ \\
\hline 20 & 227 & 206 & 205.5290 & 209.945 & 215.5516 \\
\hline 21 & 223 & 228 & 216.4187 & 224.055 & 231.8985 \\
\hline 22 & 242 & 228 & 216.4187 & 224.055 & 243.5307 \\
\hline 23 & 239 & 244 & 216.4187 & 234.11 & 246.5969 \\
\hline 24 & 266 & 244 & 216.4187 & 244.33 & 261.0037 \\
\hline RMSE & & 15.1 & 28.7295 & 14.88 & 7.6845 \\
\hline MLTE & & $100 \%$ & $100 \%$ & $75 \%$ & $50 \%$ \\
\hline
\end{tabular}

The proposed model is compared with Huarng and Yu' model [15], Cheng et al.' model [13], and Chen and Wang' model [9]. As shown in Table 10 comparing with Huarng and Yu' model, Cheng et al.' model, and Chen and Wang' model, the MLTE results are improved by the proposed model about 50\%, 50\%, and 33.3\%, respectively. This indicates that the proposed model outperforms the comparative models significantly in reflecting fluctuations in historical data. Consequently, the forecast accuracy of specific value is improved by the proposed model about $48.3 \%, 73.3 \%$, and $49.1 \%$, respectively, in terms of RMSE, which indicates that forecast accuracy is inherently improved by the proposed model by reflecting fluctuations in historical data.

\section{Conclusion}

In contrast to conventional fuzzy time series forecasting models that are based on original fuzzy time series, a different forecasting basis, fuzzy local trend time series, which is the linguistic representation of local trend variations of original data, is provided by the proposed fuzzy local trend transform. Local trend variations, which are defined as ratios between any two consecutive data points in original time series, are thereby forecasted based on fuzzy local trend time series and the specific values are are calculated accordingly. Therefore the practicability and forecast accuracy are improved by reflecting fluctuations in historical data.

The proposed model is validated by using three typical forecasting targets. The results are evaluated by two measures from different aspects. Compared to conventional fuzzy time series models, the proposed model yields about $50 \%$ and $60 \%$ average improvement in terms of MLTE and RMSE, respectively for the three application areas. The MLTE results indicate that the proposed model outperforms conventional fuzzy time series models significantly in reflecting fluctuations in historical data, and the improved RMSE results confirm an inherent enhancement of reflection of fluctuations in historical data and hence a better forecast accuracy. 
Theoretically, the proposed fuzzy local trend transform can be integrated with any fuzzy time series model. Optimization of the parameters predefined in the proposed fuzzy local trend transform is being considered. A linguistic representation of time series data based on the proposed fuzzy local trend transform for the application to time series data mining is ongoing.

\section{Bibliography}

[1] L. A. Zadeh, Outline of a New Approach to the Analysis of Complex Systems and Decision Processes, IEEE Trans. Systems, Man, and Cybernet, Vol.3, No.1, pp.28-44, 1973.

[2] L.A. Zadeh, The Concept of a Linguistic Variable and its Application to Approximate Reasoning, Part 1, Information Sciences, Vol.8, No.3, pp.199-249, 1975.

[3] Q. Song, B. S. Chissom, Fuzzy Time Series and its Model, Fuzzy Sets and Systems, Vol.54, No.3, pp.269-277, 1993.

[4] Q. Song, B. S. Chissom, Forecasting Enrollments with Fuzzy Time Series -Part I, Fuzzy Sets and Systems, Vol.54, No.1, pp.1-9, 1993.

[5] Q. Song, B. S. Chissom, Forecasting Enrollments with Fuzzy Time Series -Part II, Fuzzy Sets and Systems, Vol.62, No.1, pp.1-8, 1994.

[6] S. M. Chen, Forecasting Enrollments based on Fuzzy Time Series, Fuzzy Sets and Systems, Vol.81, No.3, pp.311-319, 1996.

[7] K. H. Huarng, Heuristic Models of Fuzzy Time Series for Forecasting, Fuzzy Sets and Systems, Vol. 123, No.3, pp.369-386, 2001.

[8] C. H. Cheng et al, Trend-weighted Fuzzy Time Series Model for TAIEX Forecasing, Lecture Notes in Compter Science, Part III, Vol.4234, pp.469-477, 2006.

[9] S. M. Chen, N. Y. Wang, Fuzzy Forecasting based on Fuzzy-trend Logical Relationship Groups, IEEE Trans. Systems, Man, and Cybernetics-Part B: Cybernetics, Vol.40, No.5, pp.1343 - 1358, 2010.

[10] H. Y. Jung et al, Fuzzy Time Series Reflecting the Fluctuation of Historical Data, Seventh International Conference on Fuzzy Systems and Knowledge Discovery, pp.473-477, 2010.

[11] J. Dan et al, Mean local trend error and fuzzy-inference-based multicriteria evaluation for supply chain demand forecasting, Journal of Advanced Computational Intelligence and Intelligent Informatics, Vol. 15, No. 2, pp.134-144, 2011.

[12] J. R. Hwang et al, Handling Forecasting Problems Using Fuzzy Time Series, Fuzzy Sets and Systems, Vol.100, No.1-3, pp.217-228, 1998.

[13] C. H. Cheng et al, Multi-attribute Fuzzy Time Series Method based on Fuzzy Clustering, Expert Systems with Applications, Vol.34, No.2, pp.1235-1242, 2008.

[14] H. K. Yu, A Refined Fuzzy Time Series Model for Forecasting, Physica A, Vol.346, No.3/4, pp.657-681, 2005.

[15] K. H. Huarng, H. K. Yu, Ratio-based Lengths of Intervals to Improve Fuzzy Time Series Forecasting, IEEE Trans. Systems, Man, and Cybernetics-Part B: Cybernetics, Vol.36, No.2, pp.328-340, 2006. 
[16] J. C. Bezdek et al, FCM: The Fuzzy C-means Clustering Algorithm, Computers \& Geosciences, Vol.10, No.2-3, pp.191-203, 1984.

[17] J. R. Hwang et al, Handling Forecasting Problems Using Fuzzy Time Series, Fuzzy Sets and Systems, Vol.100, No.1-3, pp.217-228, 1998.

[18] A. Udechukwu et al, Discovering All Frequent Trends in Time Series, The 2004 winter international symposium on information and communication technologies, pp.1-6, 2004.

[19] C. H. Chen et al, Mining Fuzzy Frequent Trends from Time Series, Expert Systems with Applications, Vol.36, No.2, Part 2, pp.4147-4153, 2009.

[20] I. Z. Batyrshin, L. B. Sheremetov, Perception-based Approach to Time Series Data Mining, Applied Soft Computing, Vol.8, No.3, pp.1211-1221, 2008.

[21] K. H. Huarng, Effective Lengths of Intervals to Impove Forecasting in Fuzzy Time Series, Fuzzy Sets and Systems, Vol.124, No.3, pp.387-397, 2001. 